\title{
Innovative treatments for adults with obstructive sleep apnea
}

This article was published in the following Dove Press journal:

Nature and Science of Sleep

18 November 2014

Number of times this article has been viewed

\author{
Terri E Weaver ${ }^{1,2}$ \\ Michael W Calik ${ }^{1,2}$ \\ Sarah S Farabi ${ }^{1,2}$ \\ Anne M Fink ${ }^{1,2}$ \\ Maria T Galang-Boquiren ${ }^{2,3}$ \\ Mary C Kapella ${ }^{1,2}$ \\ Bharati Prasad ${ }^{2,4}$ \\ David W Carley ${ }^{1,2}$
}

'Biobehavioral Health Science Department, College of Nursing, University of Illinois at Chicago; ${ }^{2}$ Center for Narcolepsy, Sleep and Health, University of Illinois at Chicago College of Nursing, ${ }^{3}$ Department of Orthodontics, University of Illinois at Chicago College of Dentistry, ${ }^{4}$ Sleep Center, Department of Medicine, University of Illinois at Chicago College of Medicine, Chicago, IL, USA
Correspondence: Terri E Weaver University of Illinois at Chicago College of Nursing, 845 S Damen Ave MC 802, Chicago, IL 606I2-7350, USA

$\mathrm{Tel}+\mathrm{I} 70877 \mid 5623$

Fax + I 708 77| 5673

Email teweaver@uic.edu
Abstract: Obstructive sleep apnea (OSA) affects one in five adult males and is associated with significant comorbidity, cognitive impairment, excessive daytime sleepiness, and reduced quality of life. For over 25 years, the primary treatment has been continuous positive airway pressure, which introduces a column of air that serves as a pneumatic splint for the upper airway, preventing the airway collapse that is the physiologic definition of this syndrome. However, issues with patient tolerance and unacceptable levels of treatment adherence motivated the exploration of other potential treatments. With greater understanding of the physiologic mechanisms associated with OSA, novel interventions have emerged in the last 5 years. The purpose of this article is to describe new treatments for OSA and associated complex sleep apnea. New approaches to complex sleep apnea have included adaptive servoventilation. There is increased literature on the contribution of behavioral interventions to improve adherence with continuous positive airway pressure that have proven quite effective. New non-surgical treatments include oral pressure devices, improved mandibular advancement devices, nasal expiratory positive airway pressure, and newer approaches to positional therapy. Recent innovations in surgical interventions have included laser-assisted uvulopalatoplasty, radiofrequency ablation, palatal implants, and electrical stimulation of the upper airway muscles. No drugs have been approved to treat OSA, but potential drug therapies have centered on increasing ventilatory drive, altering the arousal threshold, modifying loop gain (a dimensionless value quantifying the stability of the ventilatory control system), or preventing airway collapse by affecting the surface tension. An emerging approach is the application of cannabinoids to increase upper airway tone.

Keywords: obstructive sleep apnea, innovative treatment, continuous positive airway pressure, oral appliance, nerve stimulation

\section{Introduction}

Obstructive sleep apnea (OSA) is a common problem. The second most diagnosed respiratory condition after asthma, $9 \%-28 \%$ of women and $24 \%-26 \%$ of males in the US population have apneic events at a treatable level, making this syndrome a serious public health issue. ${ }^{1}$ Untreated OSA has a profound effect on health and behavior, resulting in excessive daytime sleepiness, impaired cognition and mentation, decreased daytime function, and alterations in mood. ${ }^{1}$ OSA contributes to increased heart disease, stroke, hypertension, diabetes, and obesity, with a high rate of comorbidity. The economic burden of untreated OSA is enormous, having a cost similar in magnitude to diabetes, ie, 132 billion dollars in 2002, with an estimated $\$ 15.9$ billion for the 800,000 crashes attributed to OSA. ${ }^{2,3}$ From the perspective of the health care system, for each quality-adjusted life year, $\$ 3,354$ and $\$ 11.1$ billion in collision costs would 
be saved with successful continuous positive airway pressure (CPAP) treatment, the primary intervention for OSA for over 25 years. ${ }^{3}$ CPAP introduces a column of air that serves as a pneumatic splint for the upper airway, preventing the airway collapse that is the physiologic definition of this syndrome. Despite demonstrated efficacy and potential cost savings, the overall effectiveness of CPAP is significantly limited by poor adherence. ${ }^{4}$ This limitation has prompted the development of new approaches to produce more tolerable designs for CPAP treatment, approaches to enhance adherence, and innovations in other interventions to treat OSA. With greater understanding of the physiologic mechanisms associated with OSA, novel interventions have emerged in the last 5 years. The purpose of this article is to describe new treatments for OSA and associated complex sleep apnea.

\section{Advances in positive airway pressure treatment}

Complex sleep apnea refers to a pattern of sleep-disordered breathing that includes both obstructive apneas/hypopneas and central apneas. Specifically, this occurs in patients without daytime hypercapnia. Central apneas are noted during non-rapid eye movement sleep and may "emerge" during CPAP titration. It is likely that these individuals have a high loop gain (a dimensionless value quantifying the stability of the ventilatory control system) in addition to a collapsible airway. Loop gain is measured by repeatedly dropping CPAP pressure during sleep to induce prolonged hypopneas, and then returning CPAP pressure to normal. The return of normal pressure causes an overshoot of ventilation, called the "response". Loop gain is calculated by dividing "the response" by "the stimulus" (the difference between ventilation during the hypopnea and zero ventilation). A high loop gain approaching a value of 1 reflects relative instability in the ventilatory system and predisposes to recurrent apneas. Adaptive servoventilators are spontaneous-timed bilevel positive airway pressure devices that estimate the patient's minute ventilation and tidal volume and/or flow, and respond to it by delivering variable pressure support. Theoretically, this minimizes the hyperpneic phase (which precipitates central apneas). These devices provide better control of complex sleep apnea than other CPAP devices, particularly in patients with heart failure. ${ }^{6-8}$

The S9TM (ResMed Ltd, Bella Vista, Australia) is a novel CPAP device that includes a humidification system with a heated tube, reduced noise levels, and an adaptive positive airway pressure algorithm. Use of the S9 device resulted in longer average CPAP use by approximately 30 minutes after 28 days in 50 patients with established CPAP use. ${ }^{9}$ A new CPAP modality, SensAwake ${ }^{\circledR}$ (Fisher and Paykel Healthcare Ltd, Auckland, New Zealand), was developed to monitor breathing patterns and reduce pressure with awakenings. When tested in a group of 42 CPAP-naïve patients with OSA, the SensAwake modality had results similar to those of traditional CPAP. ${ }^{10}$

\section{Interventions to promote CPAP adherence}

Adherence is the major obstacle to effective CPAP treatment. A Cochrane Collaboration report reviewing 30 studies of educational, supportive, and behavioral interventions concluded that all three types of interventions had a positive impact on CPAP usage in 2,047 adults with OSA. ${ }^{11}$ There was moderate-quality evidence to support a modest increase in adherence to CPAP using short-term educational interventions and low-quality evidence to support a large increase in CPAP adherence using behavioral interventions such as cognitive behavioral therapy and motivational interviewing. Recently, Bartlett et al conducted a randomized controlled study comparing a group cognitive behavioral therapy intervention with a social interaction control on CPAP adherence. ${ }^{12}$ They found no difference between groups in CPAP adherence or self-efficacy using CPAP at 1 or 6 months. In another randomized controlled study, Deng et al compared a stage-matched intervention with usual care in newly diagnosed OSA patients. ${ }^{13}$ Participants in the intervention group received care based on their stage of behavior change. Interventions ranged from education about OSA (stage 1) to setting and attaining goals for increased CPAP use at night (stage 4). They found significant differences between groups with regard to CPAP adherence at 1 and 3 months, and in the percentage of subjects using CPAP for more than 4 hours per night. ${ }^{13}$ Sawyer et al tested a risk screening questionnaire, ie, the Index for Nonadherence to PAP, for CPAP nonadherence in a longitudinal study that included 97 subjects with newly diagnosed OSA. The final Index for Nonadherence to PAP model included 19 items with questions regarding health literacy, self-efficacy, body mass index (BMI), marriage status, presenting symptoms, and gender. The questionnaire successfully identified CPAP nonadherers and may provide a tool for early intervention development to decrease or prevent nonadherence. ${ }^{14}$ Although there is growing evidence to support educational and behavioral interventions to enhance adherence with CPAP, further studies with stronger designs and larger samples are needed. 


\section{Nasal expiratory positive airway pressure}

During the five distinct phases of the respiratory cycle, the final expiratory phase has the greatest risk of airway occlusion. At the end of expiration, there is neither positive pressure nor inspiratory phasic activation of the upper airway dilator muscles.$^{15}$ Moreover, there is significant narrowing of the upper airway during expiration preceding apnea, ${ }^{16}$ and the lowest cross-sectional of the upper airway occurs during expiration. ${ }^{17-19}$ The nasal expiratory positive airway pressure (nEPAP) device allows for low inspiratory resistance while increasing expiratory resistance to prevent upper airway collapse (see Figure 1). ${ }^{18,19}$ It comprises a single-use device containing a mechanical valve that can create high expiratory resistance, with very low inspiratory resistance applied to each nostril with an adhesive to provide a seal. ${ }^{20,21}$ There have been six clinical trials using nEPAP. ${ }^{18,20-24}$ In all studies, there was a significant decrease in apnea-hypopnea index (AHI). Four of the nEPAP studies were uncontrolled, proof-of-concept studies, ${ }^{18,20,23,24}$ one was a prospective, sham-controlled, randomized, double-blind, multicenter clinical trial, ${ }^{21}$ and another was an extension of the latter clinical trial to observe long-term treatment durability and safety. ${ }^{22}$

In the proof-of-concept studies, two used nEPAP devices of varying expiratory resistances to increase tolerability. ${ }^{20,24}$ In these proof-of-concept studies, there was a $31 \%-49 \%$ decrease in AHI. ${ }^{18,20,23,24}$ Two studies showed no difference in sleep architecture, ${ }^{18,20}$ and one study showed significant changes in sleep architec-

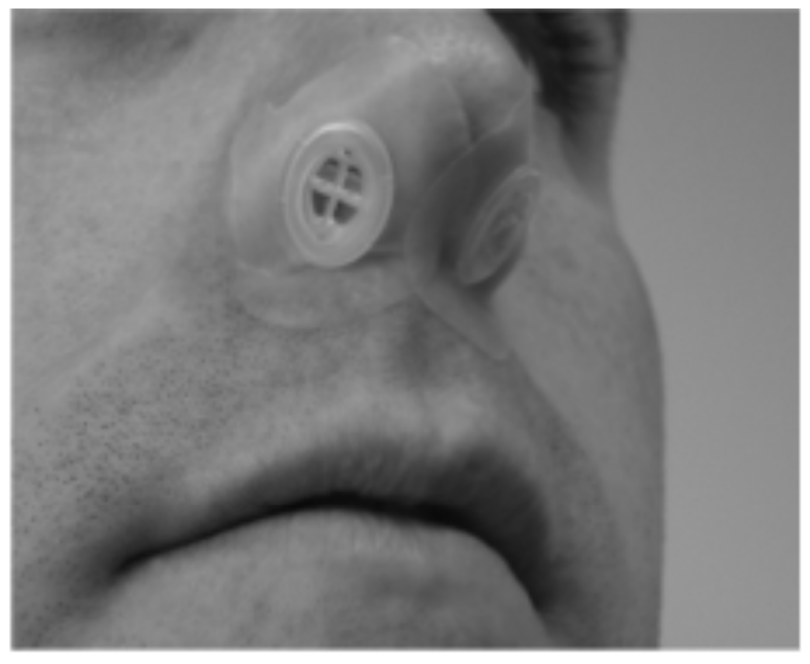

Figure I Photograph of the expiratory positive airway pressure device in place. Notes: Reprinted from Sleep Med, 12(2), Walsh JK, Griffin KS, Forst EH, et al. A convenient expiratory positive airway pressure nasal device for the treatment of sleep apnea in patients non-adherent with continuous positive airway pressure, 147-152, Copyright (C) 201 I, with permission from Elsevier. ${ }^{24}$ ture. ${ }^{24}$ Two studies quantified and showed significant improvements in daytime sleepiness or sleep quality, ${ }^{20,24}$ and three studies quantified and showed significant improvement in oxygen saturation. ${ }^{8,20,24}$ The results of the proof-of-concept trials are encouraging. However, as with CPAP, ${ }^{25}$ tolerability and adherence to nEPAP was an issue. Although self-reported use was as high as $94 \%,,^{20,24}$ $15 \%$ of participants in one study failed to take the device home. ${ }^{20}$ In another study, there was a dropout rate of $22 \%$ due to lack of tolerability of nEPAP, and many adverse events like difficulty breathing and sleeping, dry mouth, and nasal discomfort were reported. ${ }^{24}$

A prospective, sham-controlled, double-blind, multicenter clinical trial randomized newly diagnosed or previously diagnosed but untreated OSA patients to either nEPAP or a sham group. ${ }^{21}$ The sham treatment device was similar to the nEPAP device, with the only difference being that the sham device contained valves that did not provide expiratory resistance. The participants wore the nEPAP/sham devices for 3 months with two nonconsecutive night sleep studies a week into the study and two nonconsecutive night sleep studies at the end of the 3 month study period. The two nonconsecutive night sleep studies were done to evaluate on-device and off-device polysomnographs (randomly assigned). Significant median decreases in AHI after 1 week and 3 months of nEPAP were $53 \%$ and $43 \%$, respectively, when compared with sham. There were also significant decreases in oxygen desaturation index, Epworth Sleepiness Scale, and arousal index, and significant changes in sleep architecture between the nEPAP and sham groups. Adherence was high in both the nEPAP and sham groups; however, there was significantly less adherence in the nEPAP group than in the sham group (88.2\% versus $92.3 \%$ ). Adverse events, like difficulty exhaling, nasal discomfort, dry mouth, headache, and insomnia, were not significantly different between the two groups; however, more participants dropped out of the nEPAP group due to adverse events. A follow-up study evaluated the long-term durability of treatment response and safety of nEPAP. ${ }^{22}$ The positive results obtained from the original 3-month study ${ }^{21}$ were replicated after 12 months of wearing nEPAP. ${ }^{22}$ As in the 3-month study, adverse events were still reported. ${ }^{21,22}$

Although AHI is an important metric to quantify improvements in treatment response, another outcome that is important to the user as well as his or her sleeping partner is that of snoring. In three studies, the percentage of total sleep time spent snoring decreased significantly by $57.8 \%-74.4 \%$ with use of nEPAP, providing a quieter environment for the sleeping partner. ${ }^{18,20,22}$ 
The significant improvements in AHI, oxygen saturation, and snoring with nEPAP are very encouraging, and the improvement in snoring suggests that this treatment may be suitable for those with mild OSA. Moreover, given the challenges of traveling with a CPAP device, nEPAP may be an alternative for patients who travel frequently. As with CPAP, adherence and tolerability are an issue. However, considering the ease of application, compact design, low cost, and availability of these devices (ie, accessible without a prescription), nEPAP may become a second-line therapy to CPAP.

\section{Oral pressure therapy}

Oral pressure therapy, in which a vacuum is applied to the oropharynx to pull the soft palate forward and stabilize the tongue, has recently been studied in adult patients with OSA. ${ }^{26}$ The system, such as the Winx device (see Figure 2), includes a mouthpiece in a number of sizes, a vacuum pump, a console, and tubing. While breathing normally through the nose, continuous negative pressure in the oral cavity moves the soft palate forward into contact with the tongue to reduce obstruction. The negative pressure is isolated from the nasopharyngeal airway by the natural seal that occurs between the soft palate and tongue. In a multicenter, prospective, randomized, crossover trial, Colrain et al evaluated the safety and efficacy of an oral pressure therapy system in subjects with mild to severe OSA. ${ }^{27}$ The therapy was well tolerated and used on average for 6 hours at night. Oral pressure therapy was associated with clinically significant improvements in AHI, sleep quality and continuity, and daytime sleepiness. ${ }^{27}$ Although there is preliminary evidence to support oral pressure therapy as a therapy for OSA, additional studies with strong designs and larger samples are needed.

\section{Oral appliance innovations}

Although not a new concept, novel oral appliances, such as tongue repositioning or restraining devices, have recently been tested in people with OSA. A tongue-stabilizing device that uses suction to protrude the tongue was compared with a mandibular advancement device in a randomized crossover study of a small sample of patients recruited from a tertiary hospital sleep clinic. ${ }^{28}$ Although the AHI was significantly improved with both the mandibular advancement device and the tongue-stabilizing device, compliance was poor for the tongue-stabilizing device, with participants preferring the mandibular advancement device. A full breath solution appliance was examined in adults diagnosed with OSA. This is an oral appliance that has a horizontal transpalatal bar with a vertical extension called the posterior tongue restrainer.

\section{A}
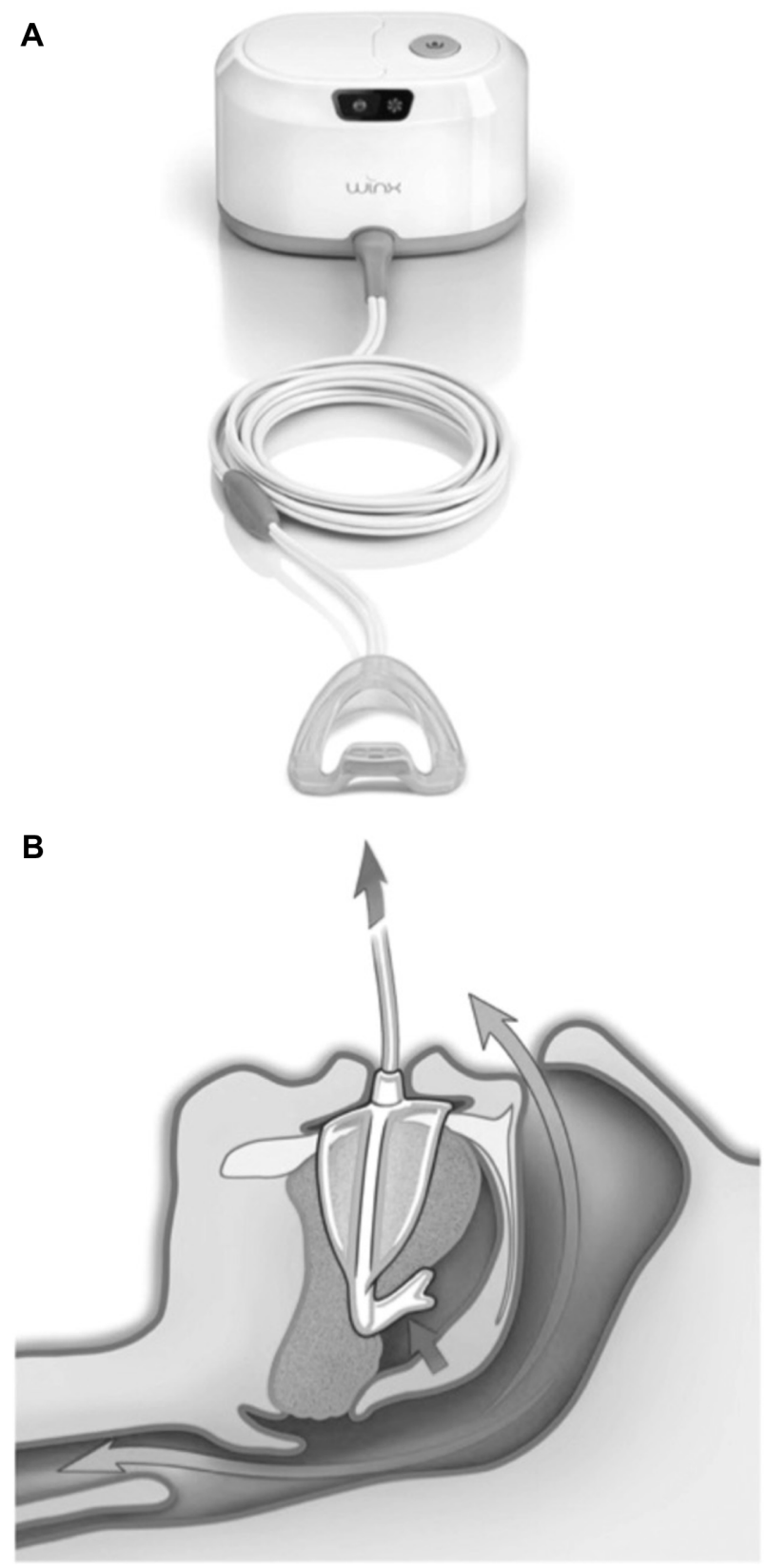

Figure 2 (A) Winx device, an oral pressure device that uses gentle suction to anteriorly and superiorly displace the tongue and the soft palate. (B) Effect of oral pressure device on the tongue and soft palate displacement.

Notes: Copyright (c) 2013. Reproduced with permission from the American College of Chest Physicians. Park JG, Morgenthaler TM, Gay PC. Novel and emerging nonpositive airway pressure therapies for sleep apnea. Chest. 20I3; 144(6): 1946-1952. ${ }^{19}$

The transpalatal bar and tissue make contact above the tongue and below the palatal mucosa when the person is supine during sleep, theoretically restraining and inhibiting the tongue from obstructing the upper airway. In this preliminary study, the mean AHI fell by $73 \%$ and the mean $\mathrm{SaO}_{2}$ significantly improved. ${ }^{29}$ Dort and Remmers tested a combined approach using a mandibular repositioning appliance with an attached tongue-retaining device; the desired 
response (respiratory disturbance index $<10$ events per hour) was achieved in $34 \%$ of subjects, but many subjects $(39 \%)$ were nonresponders. ${ }^{30}$ The authors concluded that patients require several weeks to acclimate to the device and achieve an optimal response, which may explain the poor response rate in this study.

Ngiam and Kyung investigated a novel mandibular advancement device that involved mini-implants and a facemask as anchors. ${ }^{31}$ This device may be particularly beneficial in cases where there are too few teeth to retain the oral appliances, or in patients who cannot tolerate a bulky intraoral device. Albeit based on only ten subjects, the results showed a significant reduction in AHI and snoring frequency, although design alterations in the face pads were necessary to avoid pressure sores. Almeida et al showed how short-term oral appliances could be a viable treatment option in patients who are adherent to CPAP but need to pause use of the machine in certain circumstances, such as traveling or lack of power supply. ${ }^{32}$ Having both treatment options provided good outcomes and more convenience for the patients.

One factor enhancing the effectiveness of mandibular advancement device treatment is the ability to titrate the appliance to a patient's specific needs. Titration protocols may be based on subjective parameters including patient comfort, objective parameters such as polysomnographic data, or both. To date, no consensus has been reached on a standardized titration protocol. ${ }^{33}$

In order to measure the overall therapeutic effectiveness of an oral appliance completely, objective compliance data must be obtained. To date, most relevant studies have relied on self-reporting. A thermosensor embedded in an oral appliance has been reported to have a high correlation with corresponding subjective reporting. ${ }^{34}$ This same method was studied long term, and after 1 year the correlation remained the same with respect to self-report. ${ }^{35}$ Although not currently in widespread clinical use, this provides a safe and effective method for obtaining objective reports on patient adherence. Further studies need to be conducted to determine the optimal battery life, power consumption, and storage capacity of such compliance trackers. Recently, Smith and Verrett investigated a novel device that included a supplementary sensor to enable conservation of power and memory. ${ }^{36}$ Their study also found a high correlation between subjective compliance reporting and data from their objective monitor. Objective compliance monitoring will be very valuable for long-term studies of oral appliance efficacy as well as interpreting outcomes of treatment.

\section{Prediction of oral appliance treatment outcomes}

Although oral appliance use for treating OSA has been studied extensively, it remains difficult to predict which patients will respond favorably to treatment with an oral appliance. ${ }^{37}$ Moreover, there is a need for prospective long-term studies to evaluate success rates. ${ }^{37}$ Oropharyngeal crowding and obesity were studied as predictive factors for response to oral appliance therapy. Patients with moderate OSA who have oropharyngeal crowding, identified via Mallampati scoring, and who are obese were identified as poor responders to oral appliance therapy. ${ }^{38}$ Additional research has been conducted on craniofacial morphologic features as predictors for oral appliance treatment. Cephalometric measurements such as increase in mandibular plane to cranial base angle are related to poor response to oral appliance treatment, whereas shorter soft palate length, increased cranial base angulation, minimal retroglossal airway, short anterior face height, and mandibular retrusion have been related to successful oral appliance treatment. These findings support the view that successful oral appliance treatment is moderated by multiple anatomic factors. ${ }^{39,40}$ As dental professionals transition to three-dimensional imaging, further studies may be done utilizing three-dimensional landmarks and measurements to provide more accurate predictors of the outcome of oral appliance treatment. Recent studies utilizing magnetic resonance imaging, computed tomography, and conebeam computed tomography have all provided valuable airway volumetric data that are not provided by two-dimensional cephalograms alone. ${ }^{41-43}$ Future research using volumetric airway imaging may provide valuable insights into predicting individual responses to oral appliance therapy.

Another method that can evaluate lateral airway dimensions aside from anteroposterior ones is nasal endoscopy. This method is promising as it is able to evaluate dynamic airway changes with an oral appliance either during sleep or wakefulness. ${ }^{44,45}$ In 2014, Sasao et al evaluated a number of patients diagnosed with sleep apnea and subjected them to a nasoendoscopic procedure while advancing the mandible. His group proposed conducting this test during wakefulness due to its clinical practicality compared with performing it overnight during sleep. They found that the orohypopharynx widened in all patients but the velopharynx only widened in approximately $75 \%$ of subjects, and that after oral appliance treatment, the AHI scores of those without velopharyngeal widening did not decrease significantly. The use of nasoendoscopy with mandibular advancement may be valuable in the future to evaluate velopharynx widening as a predictor of the efficacy of treatment with an oral appliance. ${ }^{45}$ 


\section{Positional therapy}

Sleep apnea in over half of patients with OSA is positiondependent, ${ }^{46}$ defined as having at least double the $\mathrm{AHI}$ in the supine position compared with the lateral position. ${ }^{47}$ Studies of the effect of positional therapy on OSA have shown positive results, ie, lower efficacy than CPAP, but often with better adherence, despite being potentially uncomfortable. ${ }^{48}$ Recent studies examining the efficacy of position therapy based on position monitoring with a supine alarm ${ }^{49}$ and a sleep position trainer device worn around the chest ${ }^{50}$ showed that these new devices were well tolerated and markedly reduced supine sleeping time and AHI. Additional studies of improved positional therapy devices are indicated.

\section{Surgical treatment approaches}

Upper airway, facial skeletal, and bariatric surgeries for morbid obesity continue to offer important alternative treatment options for adult OSA. Surgical treatments may be selected based on patient preference and ability to tolerate or adhere to CPAP. Upper airway evaluation with a clinical staging system or dynamically with endoscopy during sleep/sedation, and with technologies such as cine magnetic resonance imaging, offer ways to localize upper airway anatomic compromise and tailor treatment to individual patients. ${ }^{51}$ Nasal surgery (eg, septoplasty, turbinectomy) can improve tolerability of CPAP therapy in selected patients with OSA and reduce snoring. Minimally invasive surgeries such as palatal implants and radiofrequency or laser-assisted uvulopalatopharyngoplasty are not efficacious in adult OSA and may be considered for primary snoring. ${ }^{52}$

Many adults do not respond to single operative interventions and benefit from planned multilevel upper airway surgical modification either simultaneously or in staged protocols. ${ }^{53,54}$ A common staged protocol involves utilizing uvulopalatopharyngoplasty with tongue suspension or with genioglossus advancement and hyoid myotomy (Phase I) ${ }^{55}$ and maxillary and mandibular advancement osteotomy (Phase II). Several new techniques of upper airway surgical modifications have recently been described. Submucosal lingualplasty in combination with palatal surgery was found to be effective in improving symptoms and $\mathrm{AHI}$ in a case series of OSA patients with macroglossia. ${ }^{56} \mathrm{~A}$ transcervical technique to access the retrolingual space and perform radiofrequency ablation of hypertrophic lingual tonsils under sedation and local anesthesia has been reported and its effectiveness remains to be established. ${ }^{57}$ Other newly developed surgical approaches include modified uvulopalatopharyngoplasty and coblation channeling of the tongue, a coblation-assisted Lewis and MacKay operation, and transpalatal advancement. ${ }^{58,59}$ An investigational technique, typically used within a staged surgical approach, is transoral robotic surgery for tongue base reduction. ${ }^{60}$

Mandibular advancement osteotomy results in a permanent increase in volume and reduction in length of the upper airway, and the extent of the anterior movements of the maxilla, soft palate, and hyoid are correlated with improvement in AHI. ${ }^{61}$ The predictability of post-surgical airway change can be improved with virtual surgical planning, a technique that combines computed tomography imaging with software reconstruction and surgical expertise. ${ }^{62}$ Notwithstanding effects on AHI and symptoms, upper airway soft tissue and skeletal surgical interventions may improve quality of life, driving performance, and mortality rates in appropriately selected adults with OSA. ${ }^{63-66}$

\section{Electrical stimulation}

Remmers et al first suggested in 1978 that pharyngeal obstruction was related to loss of genioglossus muscle activity in OSA patients during sleep ${ }^{67}$ Since then, several experimental methods to treat OSA have focused on electrically stimulating upper airway muscle activity directly by transcutaneous, intraoral, or intramuscular electrodes (see Figure 3) ${ }^{68-72}$ Although these methods decreased the severity of OSA, they also had the unfortunate consequence of induced arousals and concomitant sleep fragmentation related to the electrical stimuli.

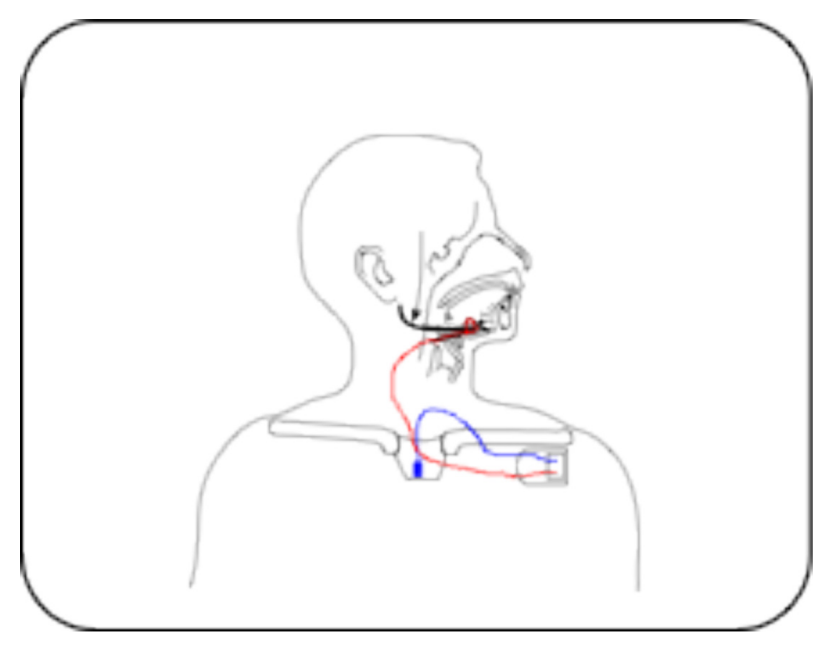

Figure 3 Schematic representation of the Inspire ${ }^{\mathrm{TM}}$ I system for hypoglossal nerve stimulation in patients with obstructive sleep apnea. The stimulator (implantable pulse generator) is connected to the respiratory sensor by means of a sensor lead. A stimulation lead connects the stimulator to the half-cuff electrode around the hypoglossal nerve.

Notes: Reprinted from J Sleep Res, 23(I), Kezirian EJ, Goding GS Jr, Malhotra A, et al, Hypoglossal nerve stimulation improves obstructive sleep apnea: I2-month outcomes, 77-83, Copyright (C) 2014, with permission from Elsevier. ${ }^{78}$ 
A more promising method of stimulating upper airway muscles to treat OSA, which emerged over a decade ago but stalled due to lack of reliable devices, ${ }^{73-76}$ is hypoglossal nerve stimulation (HNS). ${ }^{77-81}$ In HNS, a silicone cuff with stimulating electrodes is surgically placed around a unilateral hypoglossal nerve, which includes motor neurons innervating both protrusor and retractor muscles of the tongue. Appropriate placement of the electrode cuff for activation of the protrusor muscles is confirmed by observing tongue protrusion during stimulation and by electromyographic monitoring during surgery. Stimulating leads are tunneled subplatysmally via the neck to the neurostimulator, which is placed subcutaneously inferior to the clavicle. From the neurostimulator, sensory leads are tunneled subcutaneously to intercostal muscles to monitor respiration. ${ }^{77-81}$

The neurostimulator delivers either synchronous ${ }^{77-80}$ or continuous stimulation. ${ }^{81}$ In synchronous stimulation, sensory leads detect inspiratory efforts that are analyzed by the neurostimulator. The neurostimulator's software algorithm predicts the onset of inspiratory effort, delivering stimulation pulses between the end of the expiratory effort and the beginning of the next expiratory phase of each respiratory cycle. Synchronous stimulation systems depolarize the entire hypoglossal nerve during inspiratory effort, causing bulk tongue protrusion. ${ }^{76-80}$ In continuous stimulation, electrodes placed around the hypoglossal nerve are continuously and sequentially cycled from one electrode to the next in a predetermined fashion during the entire respiratory cycle. The focus of continuous stimulation is to increase tonic, rather than phasic, activation of the genioglossus. ${ }^{76,81}$ In both systems, stimulation periods are maintained on a duty cycle of $<50 \%$ to prevent neuromuscular fatigue of the tongue. ${ }^{76}$ After patients are implanted, stimulus titration for the patient occurs by gradually adjusting stimulus intensity $(\mathrm{mA})$, frequency $(\mathrm{Hz})$, and pulse width $(\mu \mathrm{sec})$ to tolerable levels that consistently abolish inspiratory flow restriction during sleep. ${ }^{76}$

Five recent HNS trials had similar inclusion and exclusion criteria. Two trials included participants with moderate to severe OSA (pretreatment AHI of 20-100) and BMI $\leq 40$ and two trials included those with an AHI of 20-50 and $\mathrm{BMI} \leq 32 .{ }^{77-80}$ One trial included subjects with $\mathrm{AHI} \geq 20$ and BMI between 25 and $40 .{ }^{81}$ The upper threshold of BMI was necessary because previous investigations showed that HNS had no therapeutic effect in individuals with elevated BMI. ${ }^{78,80}$ Exclusion criteria were similar in all trials, and included prior surgery of the head or neck, pronounced anatomic abnormalities preventing effective use of HNS, $\geq 5 \%$ of central or mixed apneas, cardiovascular disease, and sleep disorders other than OSA. ${ }^{.7-81}$ Two studies further excluded patients if palatal complete concentric collapse was observed during drug-induced sleep endoscopy (DISE) because absence of palatal complete concentric collapse during DISE has been shown to predict the therapeutic success of HNS. ${ }^{34,79,80}$

In all five studies, AHI was decreased by $\geq 50 \%$ and the oxygen desaturation index significantly improved at various time points $\left(3,6\right.$, or 12 months) after implantation..$^{80}$ The apnea index and hypopnea index each were significantly decreased. ${ }^{77,78,80}$ There were significant improvements in symptoms as quantified by improvements in daily functioning, excessive daytime sleepiness, mood, sleep quality, and quality of life. ${ }^{77,78,80}$ In two trials, there were increases in sleep efficiency and positive changes in sleep architecture, while two other studies showed no differences. ${ }^{77,78,80,81}$ When OSA patients were taken off HNS therapy for at least 5 days, the AHI and oxygen desaturation index returned back to levels prior to implantation. ${ }^{79}$ However, there is evidence that onenight off-stimulation resulted in a similar AHI and oxygen desaturation index as the previous on-stimulation night, suggesting that HNS therapy could have residual effects lasting for at least one night. ${ }^{82}$ This insight suggests the intriguing possibility of alternate night treatment. ${ }^{82}$

HNS therapy is initially invasive, requiring surgery, and adverse events related to the implantation procedure or stimulation therapy have been reported. A majority of participants reported minor adverse procedural-related events (eg, numbness/pain/swelling/infection at incision site, temporary tongue weakness) that resolved with time, pain medication, and/or antibiotic treatment, or minor adverse therapy-related events (eg, tongue abrasions cause by tongue movement over teeth, discomfort associated with stimulation) that resolved after acclimation. ${ }^{77,78,80,81}$ Serious adverse events happen infrequently and initially after implantation, and involve serious infection at the incision site requiring device removal or a subsequent surgical procedure to reposition/ replace the electrode cuff or to repair malfunctioning equipment. ${ }^{77,78,80,81}$ There was also a very small percentage of participants who had HNS devices removed due to lack of objective and subjective effectiveness. The rate of systemrelated or procedural-related serious adverse events was between $2 \%$ and $29 \%$ after implantation. ${ }^{77-80}$

Phenotyping of upper airway collapse can increase the probability of HNS success. The studies above did exclude OSA patients who had pronounced upper airway abnormalities, like enlarged tonsils or a modified Mallampati score of IV, which would prevent the success of HNS. ${ }^{77-79,81}$ 
Moreover, phenotyping for absence of palatal complete concentric collapse during DISE improved the success of HNS. ${ }^{79,80,83}$ However, the lack of uniformity in sedation methods during DISE, which carries its own risks, and the lack of an established consensus on DISE scoring systems needs to be addressed before DISE can be widely used to phenotype OSA patients for HNS. ${ }^{83}$

In view of mounting evidence for significant reductions in AHI and oxygen desaturation index, and the relatively low incidence of serious adverse events, HNS appears to be a promising alternative therapy for OSA patients with BMI $<40$ who cannot tolerate or refuse to adhere to CPAP. However, larger-scale clinical studies are needed to assess the long-term safety of HNS. In addition, it will be important to assess the efficacy and safety of HNS in subjects with more severe OSA, including comorbidities, as well as in individuals with BMI $>40$. A recent update from the Wisconsin Cohort Study has shown an increased prevalence in OSA that is correlated with an increase in BMI. ${ }^{84} \mathrm{~A}$ growing number of individuals with OSA remain at a BMI $>40$, which may limit the use of HNS as a general therapeutic alternative in OSA.

\section{Drug treatment}

Oral drugs for treating OSA have focused on five strategies, ie, increasing ventilatory drive (eg, progestagens, theophylline, acetazolamide), increasing upper airway tone (eg, serotonergics, cholinergics), decreasing rapid eye movement sleep (eg, antidepressants, clonidine), increasing arousal threshold (eg, eszopiclone), and/or increasing the cross-sectional area or reducing the surface tension of the upper airway through topical therapy (eg, fluticasone, lubricant). ${ }^{85}$ A recent Cochrane review assessed three decades of clinical trials of various drugs targeting these strategies and concluded that "some of the drugs may be helpful; however, their tolerability needs to be considered in long-term trials". ${ }^{85}$ The focus here is to present recent and innovative drug treatments for OSA.

A recent and novel approach towards increasing upper airway tone to alleviate OSA has been the use of cannabinoids. Dronabinol is a nonselective cannabinoid type $1\left(\mathrm{CB}_{1}\right)$ and type $2\left(\mathrm{CB}_{2}\right)$ receptor agonist used historically to treat anorexia/cachexia associated with acquired immunodeficiency syndrome and to control chemotherapy-induced nausea and vomiting. At doses of 2.5-10 mg per day, dronabinol significantly reduced AHI and improved subjective sleepiness in 17 adults with moderate to severe OSA, in a single-blind doseescalation proof-of-concept clinical trial. ${ }^{86}$ However, there were no changes in oxygen desaturation index, and sleep efficiency and arousal index showed improvements only during the first half of the night at the highest dose. ${ }^{86}$ A more recent secondary analysis of this study demonstrated a shift in the sleep electroencephalogram toward lower frequencies and improved ultradian cycling that was predictive of improved alertness. ${ }^{87}$ The improvement in AHI was unlikely due to any respiratory stimulant effect because animal studies have shown a decreased respiratory rate across all sleep/wake states after treatment with dronabinol. ${ }^{88}$ The authors attributed the decrease in AHI to increased upper airway tone and observed a significant improvement in supine AHI. ${ }^{86}$ This may reflect inhibition of afferent vagal nerve activity, leading to disinhibition of upper airway motor neurons. A recent report showing increased phasic activation of genioglossi, and attenuated serotonin-induced apnea in rats subjected to local nodose ganglion injections of dronabinol supports this interpretation. ${ }^{89}$ There were no serious adverse events associated with daily oral dronabinol. Common minor adverse events included evening somnolence (after drug administration and before lights out) and increased appetite. Although weight gain was not observed during this 3-week treatment period, this will need to be confirmed by longer-term trials with cannabinoids. ${ }^{86}$ Larger-scale, randomized controlled long-term studies will be needed to establish the efficacy and safety of cannabinoids in the treatment of OSA.

Another new therapeutic strategy to treat OSA is to pharmaceutically modify loop gain. ${ }^{5}$ Two studies have shown that some patients with OSA have high loop gain, and therefore, unstable ventilatory systems prone to apneas. ${ }^{90,91}$ In a recent non-blinded study, patients taking acetazolamide $500 \mathrm{mg}$ twice daily for 7 days exhibited significantly decreased loop gain and AHI when compared with baseline levels. ${ }^{92}$ This improvement in ventilatory stability was attributed to a reduction in the magnitude of the ventilatory response to arousal. The ventilatory system was desensitized to disturbances contributing to ventilatory instability. ${ }^{92}$ Unfortunately, longterm usage of acetazolamide is poorly tolerated and might not be a viable treatment option. ${ }^{5}$ Randomized, double-blind, long-term studies are needed to verify the feasibility of using acetazolamide in the treatment of OSA.

OSA has been linked to gastroesophageal reflux disease (GERD), and several studies suggest that treatment of GERD may decrease AHI and daytime sleepiness. ${ }^{93-95}$ However, these studies recruited individuals primarily for GERD. A recent study consecutively enrolled patients with OSA and investigated the effects of a proton pump inhibitor, lansoprazole, on OSA. ${ }^{96}$ Ninety-one percent of participants had pathological reflux episodes, and $22 \%$ of participants had at least $50 \%$ of apneas preceded by a reflux event during polysomnography. Three months of 
twice-daily lansoprazole decreased AHI, but there were no changes in oxygen desaturation index or sleep efficiency. Proton pump inhibitors are well tolerated, with only weak observational associations between proton pump inhibitor therapy and fractures, pneumonia, mortality, and nutritional deficiencies. ${ }^{97}$ Evaluation of GERD has the potential to become standard practice during OSA diagnosis.

The drug therapies listed above have potential in treating OSA. However, the long-term tolerability and efficacy of these drugs remain to be demonstrated. Further basic research needs to be conducted to better elucidate the neurobiological mechanisms underlying OSA; bringing fresh insights to suggest new (or to modify previous) treatment strategies in OSA. As progress is made in this area, the hope for safe and effective pharmacotherapies in OSA may become a reality.

\section{Recommendations and conclusion}

Few novel treatments have emerged that have proven as efficacious as CPAP treatment, especially for more severe disease. However, the modest level of treatment adherence makes CPAP less effective, underscoring the need for more innovative interventions. For the new approaches that have emerged, few have been robustly evaluated in randomized clinical trials and those that have had small sample sizes and limited outcomes. There is no universal definition of treatment success to guide determination of the efficacy of innovative treatments. However, outcomes that might be included to demonstrate treatment effectiveness would be adequate titration (CPAP and oral appliance), improved oxygenation, reduction in sleepiness (measured by the Epworth Sleepiness Scale), improved daily functioning (measured by the Functional Outcomes of Sleep Questionnaire), enhanced neurobehavior (metrics of sustained attention measured with the Psychomotor Vigilance Task), and improved mood (measured with a mood instrument such as the Profile of Mood States). In addition to providing the definition and outcomes of treatment success, larger clinical trials of novel interventions are needed with an evaluation of long-term treatment outcomes that reflect the variety of impairments associated with OSA.

\section{Disclosure}

Dr Carley owns common shares of Cortex Pharmaceuticals, a publicly traded company working to develop pharmacological treatments for sleep apnea syndrome and other breathing disorders, and is an inventor on patents related to pharmacological treatments for sleep apnea syndrome. The other authors report no conflicts of interest in this work.

\section{References}

1. Young T, Peppard PE, Gottlieb DJ. Epidemiology of obstructive sleep apnea: a population health perspective. Am J Respir Crit Care Med. 2002;165(9):1217-1239.

2. Al Ghanim N, Comondore VR, Fleetham J, Marra CA, Ayas NT. The economic impact of obstructive sleep apnea. Lung. 2008;186(1): 7-12.

3. Sassani A, Findley LJ, Kryger M, et al. Reducing motor-vehicle collisions, costs, and fatalities by treating obstructive sleep apnea syndrome. Sleep. 2004;27(3):453-458.

4. Weaver TE, Maislin G, Dinges DF, et al. Relationship between hours of CPAP use and achieving normal levels of sleepiness and daily functioning. Sleep. 2007;30(6):711-719.

5. Burgess KR. New insights from the measurement of loop gain in obstructive sleep apnoea. J Physiol. 2012;590 Pt 8):1781-1782.

6. Dellweg D, Kerl J, Hoehn E, Wenzel M, Koehler D. Randomized controlled trial of noninvasive positive pressure ventilation (NPPV) versus servoventilation in patients with CPAP-induced central sleep apnea (complex sleep apnea). Sleep. 2013;36(8):1163-1171.

7. Aurora RN, Chowdhuri S, Ramar K, et al. The treatment of central sleep apnea syndromes in adults: practice parameters with an evidence-based literature review and meta-analyses. Sleep. 2012;35(1):17-40.

8. Sharma BK, Bakker JP, McSharry DG, et al. Adaptive servoventilation for treatment of sleep-disordered breathing in heart failure: a systematic review and meta-analysis. Chest. 2012;142(5):1211-1221.

9. Wimms AJ, Richards GN, Benjafield AV. Assessment of the impact on compliance of a new CPAP system in obstructive sleep apnea. Sleep Breath. 2013;17(1):69-76.

10. Dungan GC 2nd, Marshall NS, Hoyos CM, Yee BJ, Grunstein RR. A randomized crossover trial of the effect of a novel method of pressure control (SensAwake) in automatic continuous positive airway pressure therapy to treat sleep disordered breathing. J Clin Sleep Med. 2011;7(3):261-267.

11. Wozniak DR, Lasserson TJ, Smith I. Educational, supportive and behavioural interventions to improve usage of continuous positive airway pressure machines in adults with obstructive sleep apnoea. Cochrane Database Syst Rev. 2014;1:CD007736.

12. Bartlett D, Wong K, Richards D, et al. Increasing adherence to obstructive sleep apnea treatment with a group social cognitive therapy treatment intervention: a randomized trial. Sleep. 2013;36(11): 1647-1654.

13. Deng T, Wang Y, Sun M, Chen B. Stage-matched intervention for adherence to CPAP in patients with obstructive sleep apnea: a randomized controlled trial. Sleep Breath. 2013;17(2):791-801.

14. Sawyer AM, King TS, Hanlon A, et al. Risk assessment for CPAP nonadherence in adults with newly diagnosed obstructive sleep apnea: preliminary testing of the Index for Nonadherence to PAP (I-NAP). Sleep Breath. March 5, 2014. [Epub ahead of print.]

15. Schwab RJ, Gefter WB. Anatomical factors: insights from imaging studies. In: Pack AI, editor. Sleep Apnea: Pathogenesis, Diagnosis, and Treatment. New York, NY, USA: Marcel Dekker; 2002.

16. Morrell MJ, Arabi Y, Zahn B, Badr MS. Progressive retropalatal narrowing preceding obstructive apnea. Am J Respir Crit Care Med. 1998;158(6):1974-1981.

17. Yucel A, Unlu M, Haktanir A, Acar M, Fidan F. Evaluation of the upper airway cross-sectional area changes in different degrees of severity of obstructive sleep apnea syndrome: cephalometric and dynamic CT study. AJNR Am J Neuroradiol. 2005;26(10):2624-2629.

18. Colrain IM, Brooks S, Black J. A pilot evaluation of a nasal expiratory resistance device for the treatment of obstructive sleep apnea. J Clin Sleep Med. 2008;4(5):426-433.

19. Park JG, Morgenthaler TM, Gay PC. Novel and emerging nonpositive airway pressure therapies for sleep apnea. Chest. 2013;144(6): 1946-1952.

20. Rosenthal L, Massie CA, Dolan DC, et al. A multicenter, prospective study of a novel nasal EPAP device in the treatment of obstructive sleep apnea: efficacy and 30-day adherence. J Clin Sleep Med. 2009;5(6): $532-537$. 
21. Berry RB, Kryger MH, Massie CA. A novel nasal expiratory positive airway pressure (EPAP) device for the treatment of obstructive sleep apnea: a randomized controlled trial. Sleep. 2011;34(4):479-485.

22. Kryger MH, Berry RB, Massie CA. Long-term use of a nasal expiratory positive airway pressure (EPAP) device as a treatment for obstructive sleep apnea (OSA). J Clin Sleep Med. 2011;7(5):449B-453B.

23. Patel AV, Hwang D, Masdeu MJ, et al. Predictors of response to a nasal expiratory resistor device and its potential mechanisms of action for treatment of obstructive sleep apnea. J Clin Sleep Med. 2011;7(1):13-22.

24. Walsh JK, Griffin KS, Forst EH, et al. A convenient expiratory positive airway pressure nasal device for the treatment of sleep apnea in patients non-adherent with continuous positive airway pressure. Sleep Med. 2011;12(2):147-152.

25. Sawyer AM, Gooneratne NS, Marcus CL, Ofer D, Richards KC, Weaver TE. A systematic review of CPAP adherence across age groups: clinical and empiric insights for developing CPAP adherence interventions. Sleep Med Rev. 2011;15(6):343-356.

26. Farid-Moayer M, Siegel LC, Black J. A feasibility evaluation of oral pressure therapy for the treatment of obstructive sleep apnea. Ther $A d v$ Respir Dis. 2013;7(1):3-12.

27. Colrain IM, Black J, Siegel LC, et al. A multicenter evaluation of oral pressure therapy for the treatment of obstructive sleep apnea. Sleep Med. 2013;14(9):830-837.

28. Deane SA, Cistulli PA, Ng AT, et al. Comparison of mandibular advancement splint and tongue stabilizing device in obstructive sleep apnea: a randomized controlled trial. Sleep. 2009;32(5):648-653.

29. Singh GD, Keropian B, Pillar G. Effects of the full breath solution appliance for the treatment of obstructive sleep apnea: a preliminary study. Cranio. 2009;27(2):109-117.

30. Dort L, Remmers J. A combination appliance for obstructive sleep apnea: the effectiveness of mandibular advancement and tongue retention. J Clin Sleep Med. 2012;8(3):265-269.

31. Ngiam J, Kyung HM. Microimplant-based mandibular advancement therapy for the treatment of snoring and obstructive sleep apnea: a prospective study. Angle Orthod. 2012;82(6):978-984.

32. Almeida FR, Mulgrew A, Ayas N, et al. Mandibular advancement splint as short-term alternative treatment in patients with obstructive sleep apnea already effectively treated with continuous positive airway pressure. J Clin Sleep Med. 2013;9(4):319-324.

33. Dieltjens M, Vanderveken OM, Heyning PH, Braem MJ. Current opinions and clinical practice in the titration of oral appliances in the treatment of sleep-disordered breathing. Sleep Med Rev. 2012;16(2): 177-185.

34. Vanderveken OM, Dieltjens M, Wouters K, et al. Objective measurement of compliance during oral appliance therapy for sleep-disordered breathing. Thorax. 2013;68(1):91-96.

35. Dieltjens M, Braem MJ, Vroegop AV, et al. Objectively measured vs self-reported compliance during oral appliance therapy for sleepdisordered breathing. Chest. 2013;144(5):1495-1502.

36. Smith Y, Verrett R. Evaluation of a novel device for measuring patient compliance with oral appliances in the treatment of obstructive sleep apnea. J Prosthodont. 2014;23(1):31-38.

37. Chen H, Lowe AA. Updates in oral appliance therapy for snoring and obstructive sleep apnea. Sleep Breath. 2013;17(2):473-486.

38. Tsuiki $\mathrm{S}$, Ito E, Isono $\mathrm{S}$, et al. Oropharyngeal crowding and obesity as predictors of oral appliance treatment response to moderate obstructive sleep apnea. Chest. 2013;144(2):558-563.

39. Shen HL, Wen YW, Chen NH, Liao YF. Craniofacial morphologic predictors of oral appliance outcomes in patients with obstructive sleep apnea. J Am Dent Assoc. 2012;143(11):1209-1217.

40. Ng AT, Darendeliler MA, Petocz P, Cistulli PA. Cephalometry and prediction of oral appliance treatment outcome. Sleep Breath. 2012;16(1):47-58.

41. Choudhury M, Padmanabhan TV. A preliminary report on the effect of a mandibular advancement device on obstructive sleep apnea using magnetic resonance imaging and polysomnography. Int J Prosthodont. 2012;25(6):613-618.
42. Furuhashi A, Yamada S, Shiomi T, et al. Effective three-dimensional evaluation analysis of upper airway form during oral appliance therapy in patients with obstructive sleep apnoea. J Oral Rehabil. 2013;40(8):582-589.

43. Alsufyani NA, Al-Saleh MA, Major PW. CBCT assessment of upper airway changes and treatment outcomes of obstructive sleep apnoea: a systematic review. Sleep Breath. 2013;17(3):911-923.

44. Johal A, Battagel JM, Kotecha BT. Sleep nasendoscopy: a diagnostic tool for predicting treatment success with mandibular advancement splints in obstructive sleep apnoea. Eur J Orthod. 2005;27(6): 607-614.

45. Sasao Y, Nohara K, Okuno K, Nakamura Y, Sakai T. Videoendoscopic diagnosis for predicting the response to oral appliance therapy in severe obstructive sleep apnea. Sleep Breath. February 15, 2014. [Epub ahead of print.]

46. Oksenberg A, Silverberg DS, Arons E, Radwan H. Positional vs nonpositional obstructive sleep apnea patients: anthropomorphic, nocturnal polysomnographic, and multiple sleep latency test data. Chest. 1997;112(3):629-639.

47. Cartwright RD. Effect of sleep position on sleep apnea severity. Sleep. 1984;7(2):110-114

48. Ravesloot M, van Maanen J, Dun L, de Vries N. The undervalued potential of positional therapy in position-dependent snoring and obstructive sleep apnea-a review of the literature. Sleep Breath. 2013;17(1):39-49.

49. Bignold JJ, Mercer JD, Antic NA, McEvoy RD, Catcheside PG. Accurate position monitoring and improved supine-dependent obstructive sleep apnea with a new position recording and supine avoidance device. J Clin Sleep Med. 2011;7(4):376-383.

50. van Maanen JP, Meester KA, Dun LN, et al. The sleep position trainer: a new treatment for positional obstructive sleep apnoea. Sleep Breath. 2013;17(2):771-779.

51. Friedman M, Ibrahim H, Joseph NJ. Staging of obstructive sleep apnea/ hypopnea syndrome: a guide to appropriate treatment. Laryngoscope. 2004;114(3):454-459.

52. Aurora RN, Casey KR, Kristo D, et al. Practice parameters for the surgical modifications of the upper airway for obstructive sleep apnea in adults. Sleep. 2010;33(10):1408-1413.

53. Caples SM, Rowley JA, Prinsell JR, et al. Surgical modifications of the upper airway for obstructive sleep apnea in adults: a systematic review and meta-analysis. Sleep. 2010;33(10):1396-1407.

54. Lin HC, Friedman M, Chang HW, Gurpinar B. The efficacy of multilevel surgery of the upper airway in adults with obstructive sleep apnea/ hypopnea syndrome. Laryngoscope. 2008;118(5):902-908.

55. Handler E, Hamans E, Goldberg AN, Mickelson S. Tongue suspension: an evidence-based review and comparison to hypopharyngeal surgery for OSA. Laryngoscope. 2014;124(1):329-336.

56. Gunawardena I, Robinson S, MacKay S, et al. Submucosal lingualplasty for adult obstructive sleep apnea. Otolaryngol Head Neck Surg. 2013;148(1):157-165.

57. Blumen M, Coquille F, Chabolle F. Lingual tonsil reduction in OSA: transcervical radiofrequency ablation. Eur Ann Otorhinolaryngol Head Neck Dis. 2012;129(6):339-342.

58. Shine NP, Lewis RH. Transpalatal advancement pharyngoplasty for obstructive sleep apnea syndrome: results and analysis of failures. Arch Otolaryngol Head Neck Surg. 2009;135(5):434-438.

59. MacKay SG, Carney AS, Woods C, et al. Modified uvulopalatopharyngoplasty and coblation channeling of the tongue for obstructive sleep apnea: a multi-centre Australian trial. J Clin Sleep Med. 2013;9(2):117-124.

60. Lin HS, Rowley JA, Badr MS, et al. Transoral robotic surgery for treatment of obstructive sleep apnea-hypopnea syndrome. Laryngoscope. 2013;123(7):1811-1816.

61. Hsieh YJ, Liao YF, Chen NH, Chen YR. Changes in the calibre of the upper airway and the surrounding structures after maxillomandibular advancement for obstructive sleep apnoea. Br J Oral Maxillofac Surg. 2014;52(5):445-451. 
62. Barrera JE. Virtual surgical planning improves surgical outcome measures in obstructive sleep apnea surgery. Laryngoscope. 2014;124(5): 1259-1266.

63. Camacho M, Certal V, Brietzke SE, et al. Tracheostomy as treatment for adult obstructive sleep apnea: a systematic review and meta-analysis. Laryngoscope. 2014;124(3):803-811.

64. Weaver EM, Woodson BT, Yueh B, et al. Studying Life Effects and Effectiveness of Palatopharyngoplasty (SLEEP) study: subjective outcomes of isolated uvulopalatopharyngoplasty. Otolaryngol Head Neck Surg. 2011;144(4):623-631.

65. Haraldsson PO, Carenfelt C, Lysdahl M, Tornros J. Long-term effect of uvulopalatopharyngoplasty on driving performance. Arch Otolaryngol Head Neck Surg. 1995;121(1):90-94.

66. Boyd SB, Walters AS, Song Y, Wang L. Comparative effectiveness of maxillomandibular advancement and uvulopalatopharyngoplasty for the treatment of moderate to severe obstructive sleep apnea. J Oral Maxillofac Surg. 2013;71(4):743-751.

67. Remmers JE, deGroot WJ, Sauerland EK, Anch AM. Pathogenesis of upper airway occlusion during sleep. J Appl Physiol Respir Environ Exerc Physiol. 1978;44(6):931-938.

68. Decker MJ, Haaga J, Arnold JL, Atzberger D, Strohl KP. Functional electrical stimulation and respiration during sleep. J Appl Physiol. 1993;75(3):1053-1061.

69. Edmonds LC, Daniels BK, Stanson AW, Sheedy PF 3rd, Shepard JW Jr. The effects of transcutaneous electrical stimulation during wakefulness and sleep in patients with obstructive sleep apnea. Am Rev Respir Dis. 1992;146(4):1030-1036

70. Guilleminault C, Powell N, Bowman B, Stoohs R. The effect of electrical stimulation on obstructive sleep apnea syndrome. Chest. 1995;107(1):67-73.

71. Miki H, Hida W, Chonan T, Kikuchi Y, Takishima T. Effects of submental electrical stimulation during sleep on upper airway patency in patients with obstructive sleep apnea. Am Rev Respir Dis. 1989;140(5):1285-1289.

72. Steier J, Seymour J, Rafferty GF, et al. Continuous transcutaneous submental electrical stimulation in obstructive sleep apnea: a feasibility study. Chest. 2011;140(4):998-1007.

73. Eisele DW, Schwartz AR, Smith PL. Tongue neuromuscular and direct hypoglossal nerve stimulation for obstructive sleep apnea. Otolaryngol Clin North Am. 2003;36(3):501-510.

74. Ilomaki J, Baer GA, Karhuketo T, Talonen P, Puhakka H. Pharyngeal patency caused by stimulation of the hypoglossal nerve in anaesthesiarelaxed patients. Acta Otolaryngol Suppl. 1997;529:210-211.

75. Oliven A, O'Hearn DJ, Boudewyns A, et al. Upper airway response to electrical stimulation of the genioglossus in obstructive sleep apnea. J Appl Physiol. 2003;95(5):2023-2029.

76. Schwartz AR, Smith PL, Oliven A. Electrical stimulation of the hypoglossal nerve: a potential therapy. J Appl Physiol. 2014;116(3):337-344.

77. Eastwood PR, Barnes M, Walsh JH, et al. Treating obstructive sleep apnea with hypoglossal nerve stimulation. Sleep. 2011;34(11):1479-1486.

78. Kezirian EJ, Goding GS Jr, Malhotra A, et al. Hypoglossal nerve stimulation improves obstructive sleep apnea: 12-month outcomes. J Sleep Res. 2014;23(1):77-83.

79. Strollo PJ Jr, Soose RJ, Maurer JT, et al. Upper-airway stimulation for obstructive sleep apnea. N Engl J Med. 2014;370(2):139-149.

80. Van de Heyning PH, Badr MS, Baskin JZ, et al. Implanted upper airway stimulation device for obstructive sleep apnea. Laryngoscope. 2012;122(7):1626-1633.

Nature and Science of Sleep

\section{Publish your work in this journal}

Nature and Science of Sleep is an international, peer-reviewed, open access journal covering all aspects of sleep science and sleep medicine, including the neurophysiology and functions of sleep, the genetics of sleep, sleep and society, biological rhythms, dreaming, sleep disorders and therapy, and strategies to optimize healthy sleep. The journal welcomes
81. Mwenge GB, Rombaux P, Dury M, Lengele B, Rodenstein D. Targeted hypoglossal neurostimulation for obstructive sleep apnoea: a 1-year pilot study. Eur Respir J. 2013;41(2):360-367.

82. Rodenstein D, Rombaux P, Lengele B, Dury M, Mwenge GB. Residual effect of THN hypoglossal stimulation in obstructive sleep apnea: a disease-modifying therapy. Am J Respir Crit Care Med. 2013;187(11): $1276-1278$.

83. Vanderveken OM, Maurer JT, Hohenhorst W, et al. Evaluation of drug-induced sleep endoscopy as a patient selection tool for implanted upper airway stimulation for obstructive sleep apnea. J Clin Sleep Med. 2013;9(5):433-438.

84. Peppard PE, Young T, Barnet JH, Palta M, Hagen EW, Hla KM. Increased prevalence of sleep-disordered breathing in adults. Am J Epidemiol. 2013;177(9):1006-1014.

85. Mason M, Welsh EJ, Smith I. Drug therapy for obstructive sleep apnoea in adults. Cochrane Database Syst Rev. 2013;5:CD003002.

86. Prasad B, Radulovacki MG, Carley DW. Proof of concept trial of dronabinol in obstructive sleep apnea. Front Psychiatry. 2013;4:1.

87. Farabi SS, Prasad B, Quinn L, Carley DW. Impact of dronabinol on quantitative electroencephalogram (qEEG) measures of sleep in obstructive sleep apnea syndrome. J Clin Sleep Med. 2014;10(1): 49-56.

88. Carley DW, Paviovic S, Janelidze M, Radulovacki M. Functional role for cannabinoids in respiratory stability during sleep. Sleep. 2002;25(4):391-398.

89. Calik MW, Radulovacki M, Carley DW. Intranodose ganglion injections of dronabinol attenuate serotonin-induced apnea in Sprague-Dawley rat. Respir Physiol Neurobiol. 2014;190:20-24.

90. Eckert DJ, White DP, Jordan AS, Malhotra A, Wellman A. Defining phenotypic causes of obstructive sleep apnea. Identification of novel therapeutic targets. Am J Respir Crit Care Med. 2013;188(8): 996-1004.

91. Salloum A, Rowley JA, Mateika JH, Chowdhuri S, Omran Q, Badr MS. Increased propensity for central apnea in patients with obstructive sleep apnea: effect of nasal continuous positive airway pressure. Am J Respir Crit Care Med. 2010;181(2):189-193.

92. Edwards BA, Connolly JG, Campana LM, et al. Acetazolamide attenuates the ventilatory response to arousal in patients with obstructive sleep apnea. Sleep. 2013;36(2):281-285.

93. Bortolotti M, Gentilini L, Morselli C, Giovannini M. Obstructive sleep apnoea is improved by a prolonged treatment of gastrooesophageal reflux with omeprazole. Dig Liver Dis. 2006;38(2):78-81.

94. Senior BA, Khan M, Schwimmer C, Rosenthal L, Benninger M. Gastroesophageal reflux and obstructive sleep apnea. Laryngoscope. 2001;111(12):2144-2146.

95. Suurna MV, Welge J, Surdulescu V, Kushner J, Steward DL. Randomized placebo-controlled trial of pantoprazole for daytime sleepiness in GERD and obstructive sleep disordered breathing. Otolaryngol Head Neck Surg. 2008;139(2):286-290.

96. Ermis F, Akyuz F, Arici S, et al. Effect of proton pump inhibitor (PPI) treatment in obstructive sleep apnea syndrome: an esophageal impedance-pHmetry study. Hepatogastroenterology. 2011;58(110-111):1566-1573.

97. Reimer C. Safety of long-term PPI therapy. Best Pract Res Clin Gastroenterol. 2013;27(3):443-454.

\section{Dovepress}

original research, clinical \& epidemiological studies, reviews \& evaluations, case reports and extended reports. The manuscript management system is completely online and includes a very quick and fair peerreview system, which is all easy to use. Visit http://www.dovepress.com/ testimonials.php to read real quotes from published authors. 